\title{
Damping hydrodynamic fluctuations in microfluidic systems
}

\author{
Ali Kalantarifard, Elnaz Alizadeh Haghighi, Caglar Elbuken* \\ Institute of Materials Science and Nanotechnology, National Nanotechnology Research Center (UNAM), Bilkent University, Ankara 06800, Turkey
}

\section{H I G H L I G H T S}

- Hydrodynamic fluctuations caused by flow sources were damped.

- Microfluidic system was modelled by including on-chip and off-chip components.

- Applying hydrodynamic damping truly monodisperse droplets were obtained.

- Demonstrated that pressure pumps operate with an inherent damping mechanism.

- Obtained one of the highest droplet monodispersity values in the literature.

\section{A R T I C L E I N F O}

\section{Article history:}

Received 3 October 2017

Received in revised form 26 November 2017

Accepted 19 December 2017

Available online 20 December 2017

\section{Keywords}

Microfluidics

Fluctuation damping

Compliance

Droplets

Monodispersity

Monodispersity
G R A P H I C A L A B S T R A C T

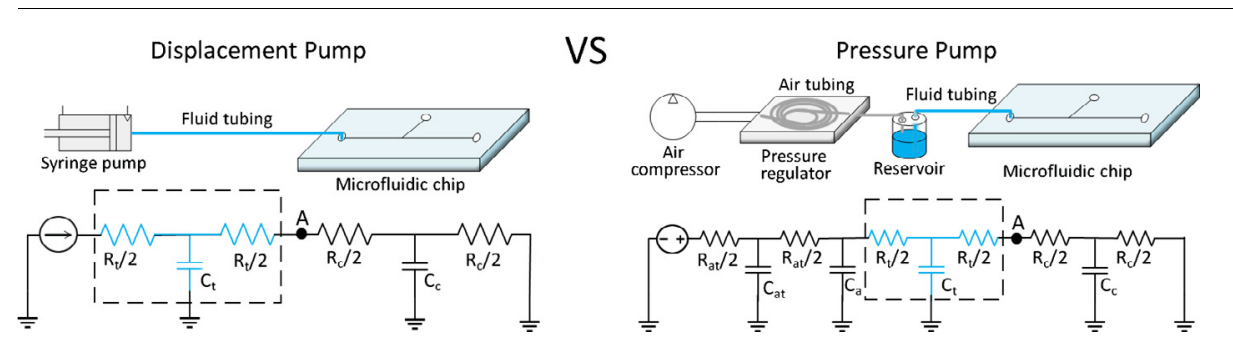

\begin{abstract}
A B S T R A C T
In this article, we report a method to damp microfluidic hydrodynamic fluctuations caused by flow sources. We demonstrate that compliance of elastomeric off-chip tubings can be used to damp fluctuations and lead to steady flow rates. We analyze the whole microfluidic system using electrical circuit analogies, and demonstrate that off-chip compliances are significant, especially for displacement pump driven systems. We apply this hydrodynamic damping method to microfluidic droplet generation. Our results show that highly monodisperse microdroplets can be obtained by syringe pump driven systems utilizing this damping effect. We reached a coefficient of variation of $0.39 \%$ for the microdroplet area using a standard T-junction geometry. Additionally, we demonstrated that pressure pumps inherently use this effect, and have so far led the high performances reported in the literature in terms of droplet monodispersity. The presented off-chip hydrodynamic damping method is not only low-cost and practical, but can also be used in elastomeric and rigid microchannels without need to introduce additional components to the fluidic circuit.
\end{abstract}

(c) 2017 Elsevier Ltd. All rights reserved.

\section{Introduction}

Microfluidic systems provide exquisite control on flow dynamics at microscale. Flow rate modulation and flow profile stability play a critical role in their performance. Hydrodynamic fluctuations deteriorate the performance of microfluidic systems that require steady flows. The fundamental source of such fluctuations

\footnotetext{
* Corresponding author.

E-mail address: elbuken@unam.bilkent.edu.tr (C. Elbuken).
}

is the flow sources. Therefore, it is necessary to develop flow sources with minimal instability or methodologies that damp the fluctuations introduced by flow sources.

Efforts have been made to analyze and minimize hydrodynamic fluctuations in order to sustain the steady state and stable fluid flow in microfluidic systems (Kang and Yang, 2012; Kim et al., 2009; Ruzicka et al., 1990). Recently, fluctuations caused by flow sources have been characterized by dimensional analysis, using the Strouhal number (Zeng et al., 2015a). It has been shown that the use of soft polydimethylsiloxane (PDMS) channels, made by increasing the base polymer/curing agent ratio, can damp 
fluctuations. In another work, flow rate fluctuations were suppressed using a PDMS microchannel that was designed to have a very thin PDMS wall as its bottom surface. This soft wall acts as a membrane and improves the monodisperse microdroplet formation (Pang et al., 2014). Furthermore, an air compliance unit and a channel with high resistance have been applied to stabilize hydrodynamic flow in microfluidic devices (Kang and Yang, 2012). Similarly, bubble-induced mechanisms have been used to damp the fluctuations (Lee et al., 2012). Although these studies demonstrate improvements in flow stability, they have several limitations. Most of these approaches are bound to elastomeric microchannels and require modification of the microchannel design, such as incorporating membrane-like surfaces or modifying channel elasticity, which can only be varied within a tight window. Softening the PDMS channels renders them susceptible to pronounced leeching and adsorption issues. In addition, incorporating additional components to the system to damp the fluctuations is not desirable for most applications that have strict microchannel design criteria.

Here, we propose a more effective and widely applicable solution to improve flow stability, by controlling off-chip compliances to minimize fluctuations due to flow sources. We utilize the tubings used to connect the flow sources to the microfluidic channels to damp the hydrodynamic fluctuations. This method provides a passive way of damping hydrodynamic fluctuations and does not require any additional components. Tubings are already utilized in most systems, unless integrated electro-osmotic pumps or capillary-driven pumping are used to drive the fluids. Therefore, the presented method is readily applicable to a wide range of microfluidic systems. Moreover, it is critical to note that hydrodynamic damping using off-chip tubings works for both elastomeric (PDMS) and rigid (acrylic, polycarbonate, glass) microfluidic devices, unlike in previous studies, which rely on the compliant components on the microchip.

We measured the hydrodynamic damping effect introduced by the tubing, which connects the flow source to the microfluidic system. The compliance of the tubing, hence the hydrodynamic damping effect, was tuned by altering the tubing length. We analyzed the fluctuations at the inlet of a microchannel and compared the improvement obtained by hydrodynamic damping when the system is run with a syringe pump or pressure pump. We demonstrate that to obtain steady flow conditions, the tubing that connects the pump to the microchip should also be considered during the design process. More importantly, we demonstrate a fundamental difference between displacement-driven and pressure-driven systems in terms of their flow stability performance. We prove that pressure pumps inherently make use of the compliance effect, which decreases the pressure fluctuation caused by the pressure regulator, thus achieving higher flow stability in most cases compared to displacement pump-driven systems (Glawdel and Ren, 2012; Korczyk et al., 2011; Zeng et al., 2015b). Since displacement pump systems lack such a damping mechanism, so far they have remained inferior. Therefore, the presented method is very effective for displacement pump-driven microfluidic systems. Through an analysis of time domain and frequency domain inlet pressure fluctuations, we demonstrate that increasing the off-chip compliance of the syringe pump-driven system significantly damps hydrodynamic fluctuations. Consequently, we show that syringe pump-driven systems are not destined to underperform on the tasks requiring fluid flow with high stability.

The method presented in this article is beneficial for applications that require fluctuation-free inlet flow rates. For example, we studied monodisperse microdroplet generation using a commonly studied T-junction geometry. It has been demonstrated in the literature that droplet monodispersity is mainly affected by external fluctuations due to unsteady flow sources (Korczyk et al., 2011; Li et al., 2014; Ruzicka et al., 1990; Zeng et al., 2015a). Another source of droplet polydispersity is the inherent fluctuations related to the generation and motion of droplets inside the channel (Beer et al., 2009; Glawdel and Ren, 2012; Van Steijn et al., 2008). Several studies have compared the droplet monodispersity of displacement pump-driven and pressure pump-driven microfluidic chips. It has repeatedly been shown that pressure pumps perform better than syringe pumps, achieving higher droplet monodispersity (Korczyk et al., 2011; Zeng et al., 2015b). The best coefficient of variation (CV) of droplet size that has been achieved using $\mathrm{T}$-junction devices is in the range of $1 \%-3 \%$ (Hwang et al., 2014; Li et al., 2015; Link et al., 2004; Pang et al., 2014; Xu et al., 2006a). We demonstrate a holistic approach in designing a microdroplet generation system, considering the resistive and compliant effects of all the system components, including off-chip components. Finally, we obtained a CV of less than $0.4 \%$ by using a syringe pump-driven system with long enough elastomeric tubing for hydrodynamic damping.

\section{Hydrodynamic damping using flexible tubing}

The proposed damping mechanism uses a flexible tubing that connects a pump to the microfluidic device, as shown in Fig. 1. Experiments have been conducted to show the performance of the flexible tubing, which functions similarly to a low-pass filter in electrical circuits. The initial experiments were performed using a syringe pump-driven flow along a PDMS microfluidic. The 5-cm long microchannel has one inlet and one outlet; the width and height of the channel is $300 \mu \mathrm{m}$ and $80 \mu \mathrm{m}$, respectively. The mold was prepared using a silicon substrate with SU-8 photoresist patterning. Sylgard 184 silicone elastomer, mixed at a weight ratio of $10: 1$ base polymer to curing agent (Dow Corning Corp), was used to fabricate the microchannels. After the bubbles were removed, the mixture was poured onto the mold and put on a hotplate at $90^{\circ} \mathrm{C}$ for $8 \mathrm{~h}$. Then, inlet/outlet holes were punched, and PDMS channel was bonded to a glass slide using $\mathrm{O}_{2}$ plasma activation. Silicone oil (SF 50) with a density and dynamic viscosity of $1000 \mathrm{~kg} / \mathrm{m}^{3}$ and $50 \mathrm{mPa}$ s was driven through the microchannel. The sample was introduced to the microchannel at a constant flow rate of $3 \mu \mathrm{l} / \mathrm{min}$ using a syringe pump (KDScientific 270).

The fluidic connection from the pump to the microchip was made using five different lengths of tubing $(5,10,20,50$ and $100 \mathrm{~cm}$ ). First, flexible silicone tubing (Cole-Parmer) with $0.8 \mathrm{~mm}$ inner diameter (I.D.) and $2.4 \mathrm{~mm}$ outer diameter (O.D.) was used. The Young's modulus and Poisson's ratio of the flexible tubing are 1.9 MPa and 0.48, respectively. Then, rigid PTFE tubing was used for comparison. The rigid tubing (Cole-Parmer) has an I.D. of $0.8 \mathrm{~mm}$ and O.D. of $1.41 \mathrm{~mm}$. Young's modulus and Poisson's ratio of the rigid tubing are $334.7 \mathrm{MPa}$ and 0.48 , respectively. A pressure sensor (Honeywell 40PC015G1A 0-15 psi) was used for continuous monitoring of pressure fluctuations caused by the flow source. The pressure sensor has a linear response with an accuracy of $0.2 \%$ and sensitivity of $266.6 \mathrm{mV} / \mathrm{psi}$. The pressure values were recorded using a data acquisition unit (NI DAQ Pad-6015). The data were processed using Matlab, and the time domain and frequency domain signals for different lengths of flexible and rigid tubings are shown in Figs. 2 and 3, respectively.

As demonstrated in Fig. 2, increasing the tubing length from 5 to $100 \mathrm{~cm}$ has a remarkable effect on fluctuation damping. Fig. 2a shows the normalized pressure values read by the pressure sensor placed at the outlet of the syringe pump. Fig. 2b shows the frequency components of the time domain signals, shown in Fig. 2a, after Fourier transform. The comparison of the time domain and frequency domain signals shows that increasing the length of flexible tubing decreases hydrodynamic pressure fluctuations. 


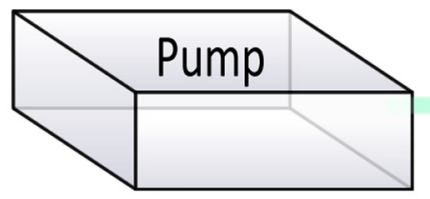

flexible tubing

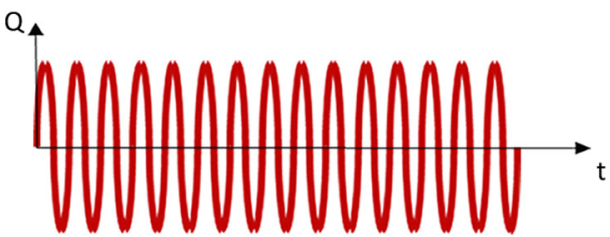

Fluctuations in the microfluidic system without flexible tubing
Microfluidic chip

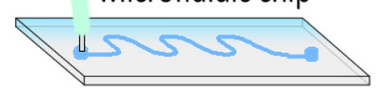

\section{Fluctuations in the microfluidic system with flexible tubing}

Fig. 1. Schematic of the microfluidic system used to test the effect of the tubing as a hydrodynamic fluctuation damping element.
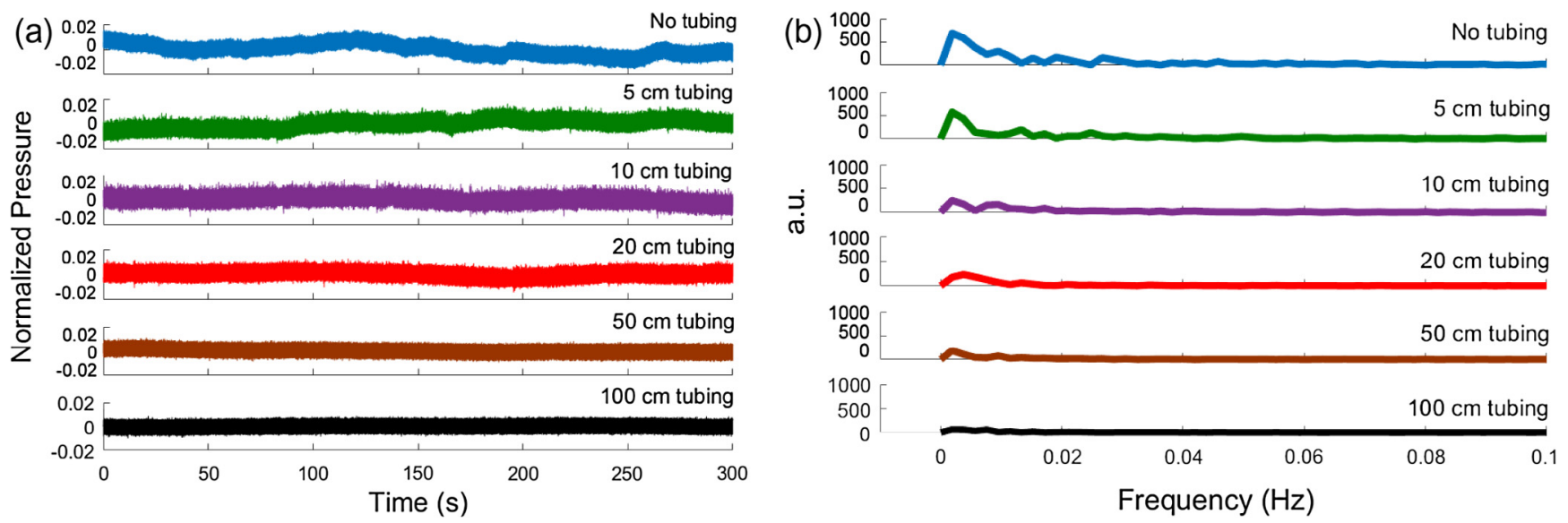

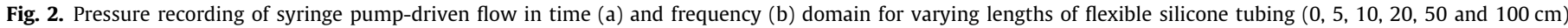
connecting the pump and the microchannel.
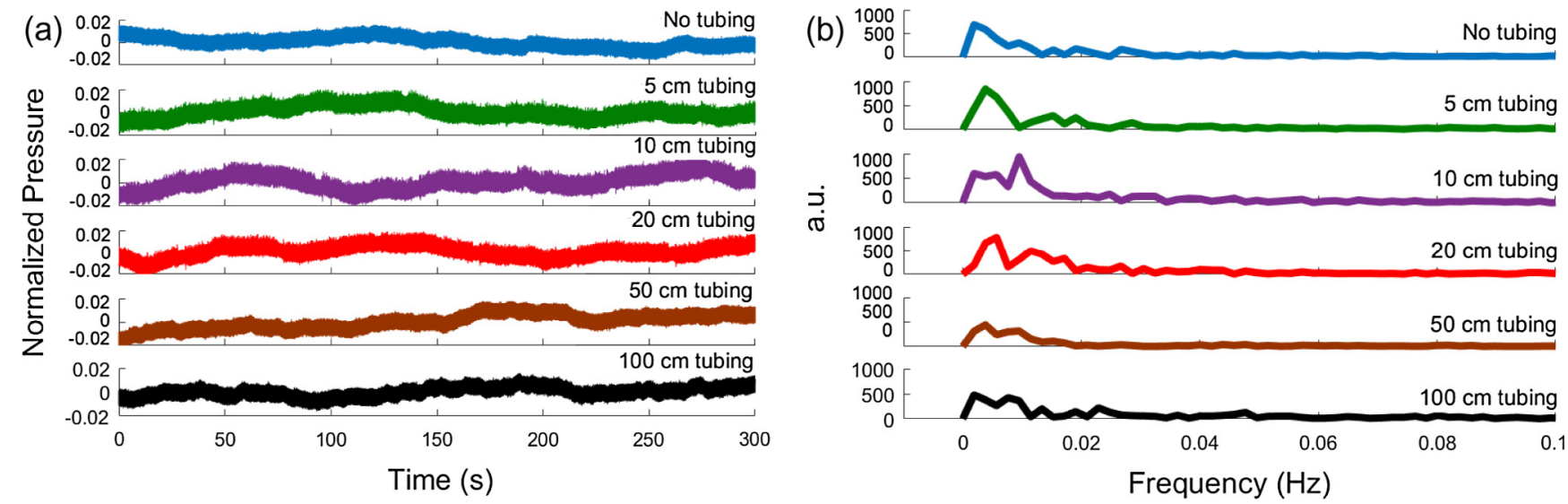

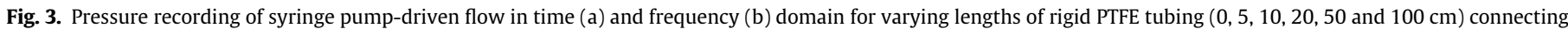
the pump and the microchannel.

As a control experiment, a similar measurement was performed using rigid PTFE tubings of the same length, as shown in Fig. 2. The results given in Fig. 3 show that almost no difference was observed in terms of pressure fluctuations when the tubing length was increased. During these measurements, rigid connectors (ColeParmer) were used to prevent additional compliance, so that only the effect of the tubing were analyzed.

\section{Damping effect for syringe pump and pressure driven system}

The development of rapid fabrication methods has enabled researchers to prototype microfluidic systems fairly easily. This sometimes leads to an oversimplification of the design process, which requires modeling of the systems parameters for demanding applications. Hydrodynamic damping and obtaining stable flow 
conditions is one of the cases that require modeling of the whole microfluidic system. Analysis of the system characteristics and optimization of each component is routinely performed for electrical circuit design. Thankfully, fluidic circuits can also be modeled using electrical circuit analogy and using the tools available for electrical engineers (Oh et al., 2012). We apply this approach to model the hydrodynamic damping effect and demonstrate the differences between syringe pump- and pressure pump-driven systems as illustrated in Fig. 4a and b, respectively. The equivalent circuit component for any flexible structure in the system, such as tubing $(t)$ or channel $(c)$, includes a resistor $\left(R_{t}, R_{c}\right)$ and a capacitor $\left(C_{t}, C_{c}\right)$ (Bruus, 2007). The resistor is placed as two half-value resistances in series, so that the compliance of the tubing is applied at mid-length. Moreover, there is capacitance for the pressure pump-driven system, due to the compressibility of the air (a) inside the reservoir $\left(C_{a}\right)$, and through the tubing in the pressure regulator chamber $\left(C_{a t}\right)$. The equivalent circuit model makes it possible to analyze the effect of different parameters such as tubing length, diameter, Young's modulus and reservoir air volume on the fluctuations due to the flow source.

The components in the electrical circuit model can be calculated analytically. The Hagen-Poiseuille Law for the laminar steady state flow of incompressible fluid at constant flow rate through a finite geometry is stated as:

$\Delta P=R_{H} Q$

where $\Delta P$ is the pressure drop, $R_{H}$ is hydrodynamic resistance and $Q$ is the flow rate along the conduit. For a cylindrical tubing, $R_{H}$ is defined as:

$R_{H}=\frac{8 \eta L}{\pi r_{i}^{4}}$

where $\eta$ is the fluid dynamic viscosity, $L$ is the tubing length, and $r_{i}$ is the tubing inner radius. For a rectangular microchannel (width $w$ and height $h$ ), with a high aspect ratio $(w>h)$, the hydrodynamic resistance can be calculated as in (Oh et al., 2012):

$R_{H}=\frac{12 \eta L}{w h^{3}\left(1-\frac{0.63 h}{w}\right)}$

The pressure change caused by the conduit flexibility or fluid compressibility results in change in the volume of the fluid along the conduit. This behavior is similar to the capacitance in the electric circuit. Therefore, the hydrodynamic capacitance is defined by the following equation:

$Q=\frac{d V}{d t}=\frac{d V}{d P} \frac{d P}{d t}=C_{h} \frac{d P}{d t}$

where $C_{h}$ is the hydrodynamic capacitance. Due to the internal pressure induced by the fluid, the shape of the flexible tubing changes in the radial direction. For the elastic thick wall tube, which is sub- jected only to internal pressure, the elastic stresses on the internal surface of the tubing are given by the Lame equations (Schmid et al., 2014).

$\sigma_{r}=-P \quad \sigma_{\theta}=\frac{\left(r_{i}^{2}+r_{o}^{2}\right) P}{\left(r_{o}^{2}-r_{i}^{2}\right)} \quad \sigma_{a}=\frac{r_{i}^{2} P}{\left(r_{o}^{2}-r_{i}^{2}\right)}$

where $\sigma_{r}, \sigma_{\theta}$ and $\sigma_{a}$, are radial stress, circumferential stress and axial stress, respectively, while $r_{i}$ and $r_{o}$ stand for inner and outer radiuses, respectively, and $P$ indicates internal pressure. Using $V=\pi r_{i}^{2} L$ and $d V=2 \pi r_{i} L . d r$, the radial strain $\left(\varepsilon_{r}\right)$ is determined as:

$\varepsilon_{r}=\frac{d r}{r_{i}}=\frac{d V}{2 \pi r_{i}^{2} L}$

where $L$ and $V$ are the length and volume of the tubing, respectively. According to Hook's law, for an isotropic material, the radial strain is proportional to the stress as follows:

$\varepsilon_{r}=\frac{1}{E}\left[\sigma_{r}-v\left(\sigma_{\theta}+\sigma_{a}\right)\right]$

where $E$ and $v$ are Young's modulus and Poisson's ratio, respectively. Using Eqs. (4)-(7), the hydrodynamic capacitance of the tubing $\left(C_{t}\right)$ is defined as:

$C_{t}=\frac{2 \pi r_{i}^{2} L\left[(1+v) r_{o}^{2}-(1-2 v) r_{i}^{2}\right]}{E\left(r_{o}^{2}-r_{i}^{2}\right)}$

which is proportional to the tubing length. In addition, the capacitance of the rectangular microchannel $\left(C_{c}\right)$ is defined as (Cartas Ayala, 2013):

$C_{c}=\frac{\alpha h w L(1+v)}{E}$

where $h, w$ and $L$ are the height, width and length of the channel, respectively. Poisson's ratio and Young's modulus of PDMS was taken as 0.5 and $2 \mathrm{MPa}$, respectively. In addition, $\alpha$ is approximately constant for a given microchannel and on the order of 1 (Cartas Ayala, 2013). In addition, there is another capacitance due to the compressed air inside the tubing and reservoir for the pressure pump-driven system. The compressibility of the air $(\beta)$ is defined as:

$\beta=-\frac{1}{V} \frac{\partial V}{\partial P}$

From Eq. (10), and due to air compression, we have:

$Q=-\frac{d V}{d t}=V\left(-\frac{1}{V} \frac{\partial V}{\partial P}\right) \frac{d P}{d t}=V \beta \frac{d P}{d t}$

Using the ideal gas law and Eqs. (10) and (11), the air capacitance $\left(C_{a}\right)$ is defined as:

$C_{a}=\frac{V}{P}$ (a)

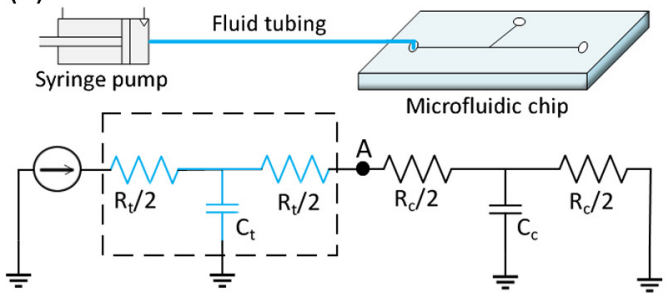

(b)

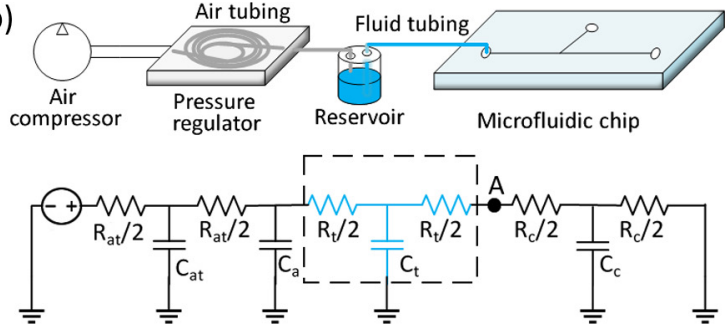

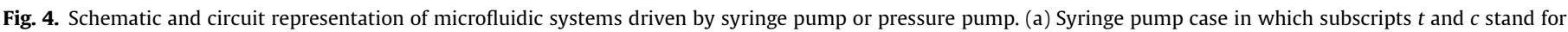

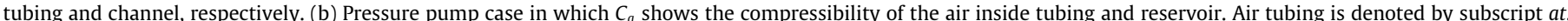

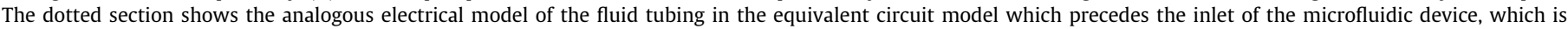
denoted by node $\mathrm{A}$. 
This analysis allows one to derive the equivalent model of a microfluidic system. Additionally, it should be noted that the flow sources in Fig. 4 were modeled as current and voltage sources for the syringe pump and pressure pump, respectively. In the previous section, we demonstrated that by using a flexible tubing to connect a syringe pump to a microfluidic channel, hydrodynamic fluctuations can be damped to varying extents, depending on the tubing length. We repeated the same experiment for a pressure pumpgenerated flow. A pressure pump (Elveflow OB1) was connected to the PDMS microchannel using flexible silicone tubing of varying lengths. Pressure was set to a constant 150 mbar, so that the mean flow rate at the microchannel was the same as in the syringe pump-driven system, explained in Fig. 2. The pressure values recorded for a time duration of $300 \mathrm{~s}$ were given in Fig. 5, in both time domain and frequency domain. As seen, there is only marginal increase in the hydrodynamic fluctuation damping with increasing tubing length. The comparison between Figs. 2 and 5 demonstrates that the damping effect of the flexible tubing is much more significant in the case of a syringe pump-driven system, compared to a pressure pump-driven system.

This observation can be explained by calculating the compliance values for the pressure-driven flow shown in Fig. $4 \mathrm{~b}$. When $100-\mathrm{cm}$ long silicone tubing was used, $C_{t}$ is $5.84 \times 10^{-13} \mathrm{~m}^{3} / \mathrm{Pa}$, whereas $C_{a t}$ and $C_{a}$ values are $5.1 \times 10^{-9}$ and $1.53 \times 10^{-9} \mathrm{~m}^{3} / \mathrm{Pa}$, respectively. Thus, the compliance effect of the tubing is negligible compared to the compliance of the air inside the fluidic system. These results clearly demonstrate that pressure pumps operate with an inherent hydrodynamic damping mechanism. Therefore, the contribution of the additional damping, due to the flexible tubing used upstream the microchannel inlet, is relatively low, depending on the pump's internal tubing parameters (length, Young's modulus, and inner diameter), the air volume left in the sample reservoir, and the supplied pressure.

\section{Monodisperse droplet generation using hydrodynamic damping}

Droplet-based microfluidics is an important class of microfluidic platforms, utilized in many biochemical analyses. Using droplet-based systems, one can carry out experiments rapidly and perform reactions in a large number of isolated droplets. Access to a large number of identical droplets enables researchers to obtain a uniform and reliable pack of data for analysis. One of the most common rationales for using droplet-based systems is the ability to form monodisperse droplets. To understand the governing physics, earlier studies on droplet monodispersity focused on controlling the parameters that contribute to droplet size such as surfactant, wettability, flow rate ratio, interfacial tension, viscosity ratio, microchannel geometry and capillary number (Garstecki et al., 2006; Glawdel et al., 2012a, 2012b; Lee et al., 2009; Raj et al., 2016; van Steijn et al., 2010; Wang et al., 2015; Xu et al., 2008, 2006a, 2006b). More recently, the contribution of flow sources to droplet formation dynamics has also been studied (Zeng et al., 2015a).

Monodispersity of microdroplets formed by microfluidic droplet generation systems is influenced by internal pressure fluctuations due to droplet formation, and external fluctuations caused by flow sources. It is critical to minimize these fluctuations, which requires a thorough analysis of the source of unsteadiness. In this section, the effect of hydrodynamic damping on droplet monodispersity in a droplet-generating system is analyzed for both displacement-driven and pressure-driven systems.

\subsection{Experimental setup}

\subsubsection{Microfluidic chip}

Experimental droplet generation was performed using a PDMS T-junction microfluidic device. Fig. 6 shows the schematic of the experimental setups used for syringe pump- and pressure pumpdriven systems. There are two inlets and one outlet in the microchannel. Two immiscible fluids were driven through the microchannels to generate droplets. Silicone oil (SF 50) with density and dynamic viscosity of $1000 \mathrm{~kg} / \mathrm{m}^{3}$ and $50 \mathrm{mPa}$ s was used as the continuous fluid. Distilled water was driven into the second inlet as the dispersed phase with density and dynamic viscosity of $1000 \mathrm{~kg} / \mathrm{m}^{3}$ and $1 \mathrm{mPa} s$, respectively. The width and height of all channels were $300 \mu \mathrm{m}$ and $80 \mu \mathrm{m}$, respectively. The experiments were performed using the same microfluidic device, in order to make a fair comparison between the performance of the syringe pump and the pressure pump.

\subsubsection{Flow sources and peripheral components}

As illustrated in Fig. 6, our goal is to compare the two most commonly utilized flow sources used in microfluidic systems. For the first setting, the two immiscible fluids were introduced to the microchannel at constant flow rates using syringe pumps (KDScientific 270). Two syringe pumps were used to drive oil and water at $3 \mu \mathrm{l} / \mathrm{min}$ and $1 \mu \mathrm{l} / \mathrm{min}$, respectively. For the pressure pump case, a multi-channel pressure pump (Elveflow OB1) was used to pump oil and water into the microchannel. Using the pressure pump, we set the oil and water pressure to $95 \mathrm{mbar}$ and $55 \mathrm{mbar}$, respec-
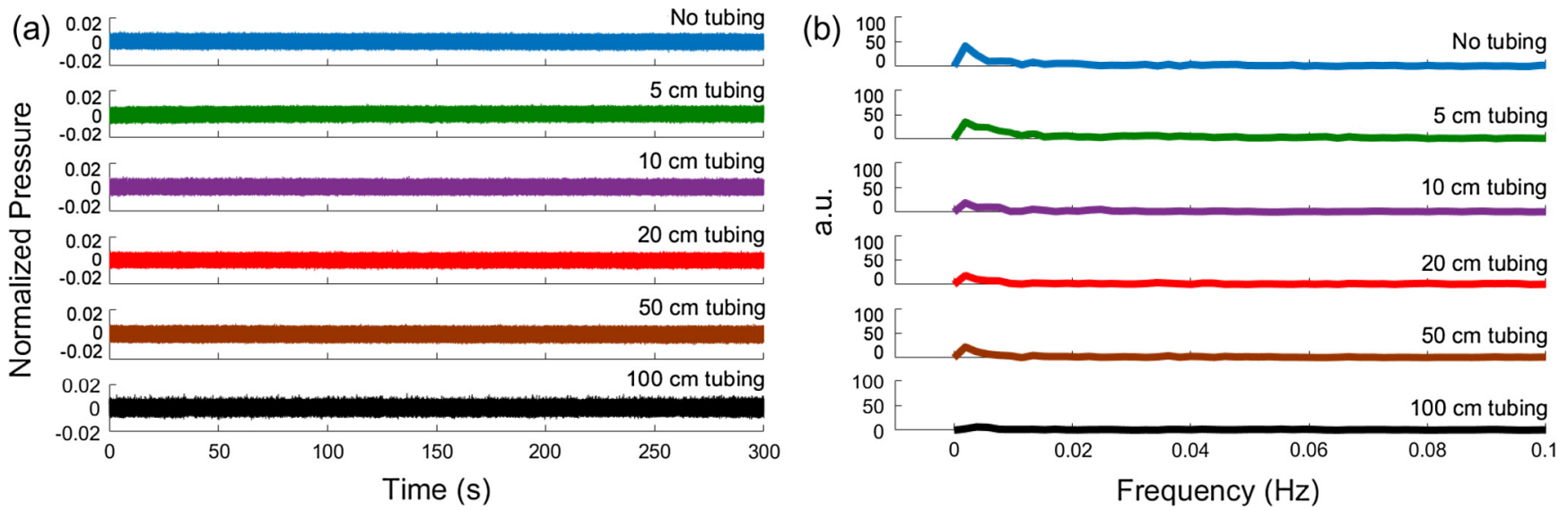

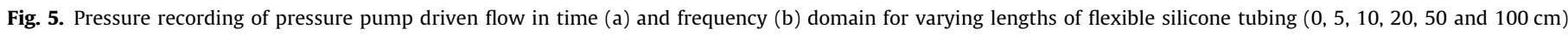
connecting the pump and the microchannel. 


\section{(a) Syringe pump}

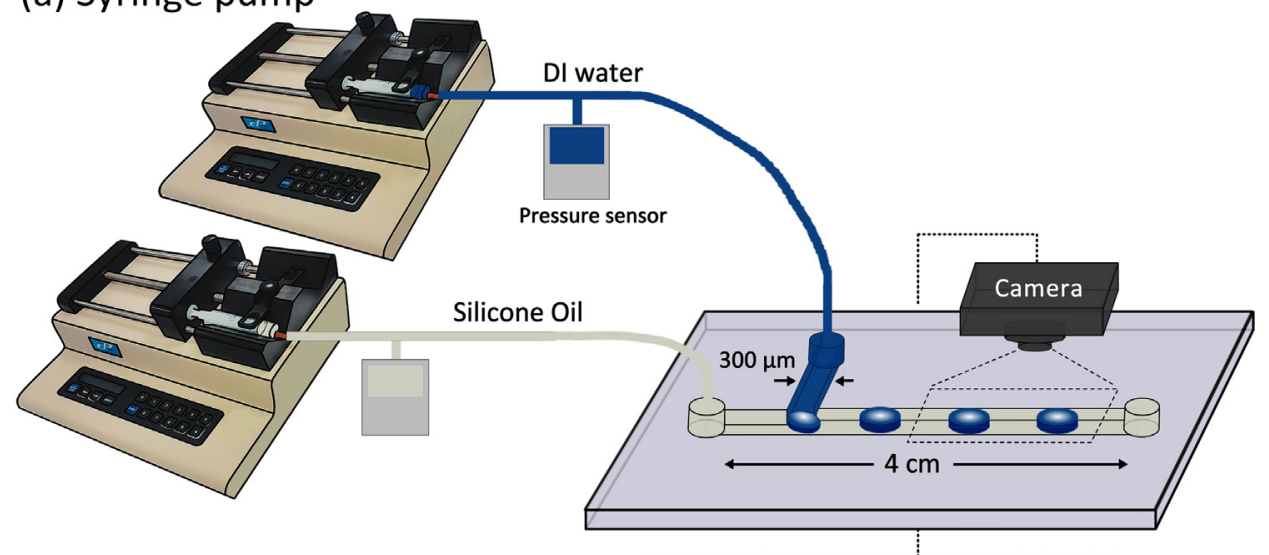

(b) Pressure pump

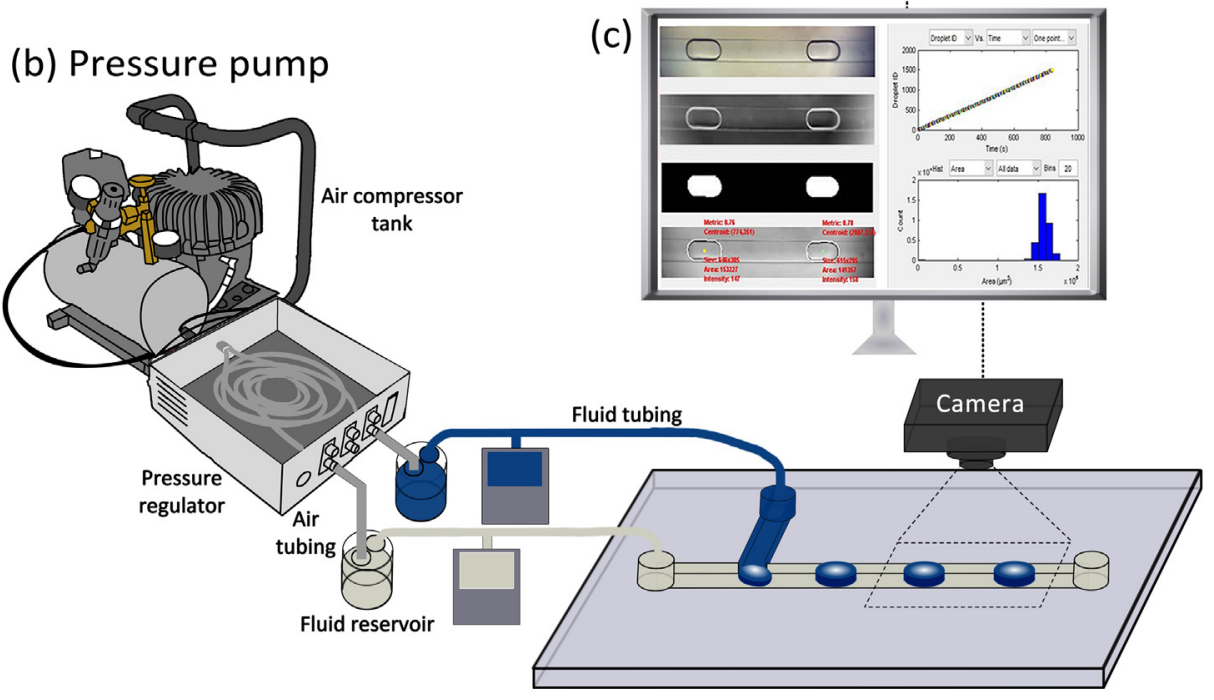

(c)

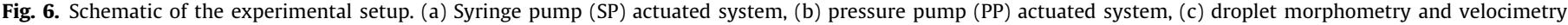
(DMV) software for calculating droplet area (Basu, 2013).

tively. In both cases, the Capillary number was kept the same to ensure that droplets were formed in the same regime.

All the fluidic connections from the pumps to the microchip were made using flexible silicone tubing, characteristics of which are mentioned in Section 2. Pressure fluctuations caused by the unsteadiness of the flow source influencing oil and water flows are measured by pressure sensors at each inlet. For hydrodynamic damping, either $20 \mathrm{~cm}$-long or $100 \mathrm{~cm}$-long elastomeric tubings were used. An inverted microscope (Omano OMFL600), equipped with a camera (Optixcam OCS-5.0), was used to capture the droplet motion using a 20x objective. As shown in Fig. 6c, for both cases, Droplet Morphometry and Velocimetry software (DMV) was used (Basu, 2013) to analyze the droplet area from the recorded videos in order to obtain the coefficient of variation (CV), which is a metric used to compare droplet monodispersity. In all the experiments the data were recorded after 5 min of running the system to ensure that steady-state flow conditions are achieved. Moreover, we backed up our analysis with numerical modeling, which allows us to study the ideal fluctuation-free inlet conditions.

\subsection{Numerical modeling}

Numerical modeling of droplet-based systems is a convenient alternative for experiments, since it allows much faster investigation and analysis of several droplet generation scenarios. Level set method (LSM) is a common method for simulating two-phase flow and droplet formation (Boy et al., 2008). Using LSM, it is possible to track time-dependent dispersed phase motion and simulate droplet generation. Change in contact angle, viscosity ratio, flow rate ratio, capillary number and interfacial tension between two immiscible fluids can be analyzed using LSM (Bashir et al., 2011).

LSM is performed using the 2D Laminar Flow Two-Phase Flow, LSM interface module of COMSOL Multiphysics 5.0. Coupling continuity Eq. (13) and incompressible Navier-Stokes Eq. (14) with the level set in Eq. (15) allowed the tracking of time-dependent dispersed phase motion and simulation of water in oil (w/o) droplet generation.

$\nabla \cdot u=0$

$\rho \frac{\partial u}{\partial t}+\rho(u \cdot \nabla) u=\nabla \cdot\left[-p I+\eta\left(\nabla u+(\nabla u)^{T}\right)\right]+F_{s t}$

$\frac{\partial \phi}{\partial t}+u . \nabla \phi=\gamma \nabla \cdot\left(\varepsilon \nabla \phi-\phi(1-\phi) \frac{\nabla \phi}{|\nabla \phi|}\right)$

where $u, \rho, t, p, \eta$ and $F_{s t}$ denote velocity $(\mathrm{m} / \mathrm{s})$, density $\left(\mathrm{kg} / \mathrm{m}^{3}\right)$, time $(\mathrm{s})$, pressure $(\mathrm{Pa})$, dynamic viscosity ( $\mathrm{Pa} \mathrm{s}$ ) and surface tension force $\left(\mathrm{N} / \mathrm{m}^{3}\right)$, respectively. Additionally, $\gamma$ and $\varepsilon$ are numerical stabilization parameters, and $\phi(x, t)$ is the level set function. The fluid interface is specified by the $\phi(x, t)=0.5$ contour of the level set function, whereas continuous and dispersed phases were modeled 
as $\phi(x, t)=0$ and $\phi(x, t)=1$, respectively. The density and dynamic viscosity are obtained using:

$\rho=\rho_{1}+\left(\rho_{2}-\rho_{1}\right) \phi$

$\eta=\eta_{1}+\left(\eta_{2}-\eta_{1}\right) \phi$

where $\rho_{1}, \rho_{2}, \eta_{1}$ and $\eta_{2}$ are densities and dynamic viscosities of fluid 1 (continuous phase) and fluid 2 (dispersed phase), respectively. Using the above equations, the formation of droplets at a Tjunction and their motion along a microchannel were simulated in 2D flow, as shown in Fig. 7.

Microdroplets were formed using two immiscible fluids, whose properties are given in Table 1 , together with the boundary conditions. For the numerical model, no wet wall boundary conditions were used.

The inlet boundary condition in simulations is determined based on the way two immiscible fluids are fed to the microfluidic device. The syringe pump is modeled as a flow rate boundary condition, whereas the pressure pump is modeled as a pressure inlet condition. Two kinds of simulations were performed in this study: with and without inlet boundary condition fluctuation. The initial values of flow rate and pressure were tailored so that the same size of droplets was obtained as in the experiments. These values are different from the experimental values, since only a fraction of the straight main channel is simulated for computational efficiency. For the fluctuation-free simulations, the inlet pressure or flow rates values were set as constant. Then, fluctuations of pressure and flow rates were taken into account and imported as inlet parameters in the numerical modeling. The equivalent circuit of each system was formed after calculating the capacitance and resistance of the corresponding components, based on the equations given in the previous section. The calculated capacitance and resistance are given in Table 2 .

The fluid tubing is the variable component in the equivalent circuit diagrams shown in Fig. 4. We used tubings of $20 \mathrm{~cm}$ and 100 $\mathrm{cm}$ in length for the experiments and the numerical model. The experimental pressure fluctuations were converted to periodic fluctuations and were then applied to the equivalent circuit, simulated with LTspice software (Linear Technology). Using the equivalent circuit, we obtained fluctuations for the continuous and dispersed phase solutions at different points in the circuit. The damped fluctuations at the inlet of the microfluidic device (node A in Fig. 4) were obtained for water and oil. Then, the periodic fluctuations were normalized and imported into the numerical simulations. All simulations were carried out using time step of $0.0005 \mathrm{~s}$
Table 1

Numerical modeling parameters.

\begin{tabular}{lll}
\hline Parameter & Fluid 1 & Fluid2 \\
\hline Density $\left(\mathrm{kg} / \mathrm{m}^{3}\right)$ & 1000 & 1000 \\
Dynamic viscosity $(\mathrm{mPa} \mathrm{s})$ & 50 & 1 \\
Flow rate for syringe pump $(\mu \mathrm{l} / \mathrm{s})$ & 0.043 & 0.0086 \\
Pressure for pressure pump $(\mathrm{Pa})$ & 245 & 78 \\
Contact angle $(\mathrm{rad})$ & $3 \pi / 4$ & \\
Slip length $(\mu \mathrm{m})$ & 1 & \\
Surface tension coefficient $(\mathrm{N} / \mathrm{m})$ & 0.005 & \\
\hline
\end{tabular}

and 2280 mesh elements. As illustrated in Fig. 7, the main channel was divided into three identical domains to calculate the droplet area. After the simulation was completed, we obtained the droplet area by calculating the volume fraction in the domain which contains the droplet as a whole using post-processing options.

\subsection{Results and discussion}

Droplet monodispersity for numerical and experimental results has been analyzed by calculating the coefficient of variation (CV) of the droplet area. DMV software was used to obtain the experimental CV of the droplet area (Basu, 2013). There are two ways of studying droplet size variation in 2D simulations: calculating droplet length, or calculating droplet area. We used droplet area calculation to obtain a better approximation to 3D. The comparison of droplet monodispersity, using syringe pump (SP) and pressure pump (PP), was performed for three cases: numerical results with inlet condition fluctuations (pressure or flow rate), numerical results without fluctuations and experimental results. We plotted the histograms of the droplet area that had been obtained experimentally and numerically in Figs. 8 and 9, respectively. As illustrated in Fig. 8, using longer tubing leads to lower CV values for the droplet area in both SP and PP cases. For the PP-driven system, approximately twofold improvement was obtained using the longer tubing. In the SP case, CV of the droplet area decreased from $4.46 \%$ to $0.388 \%$. Order of magnitude difference between CVs of 20 $\mathrm{cm}$ and $100-\mathrm{cm}$ tubing shows the significance of the hydrodynamic damping on droplet monodispersity.

Comparing the CV values of SP and PP shows that the effect of the fluid tubing length for SP is much higher than for PP. This is due to the fact that the fluid tubing is the only compliance, which damps the fluctuations for SP. However, in the PP case, in addition to fluid tubing, air tubing and the compressibility of the air inside

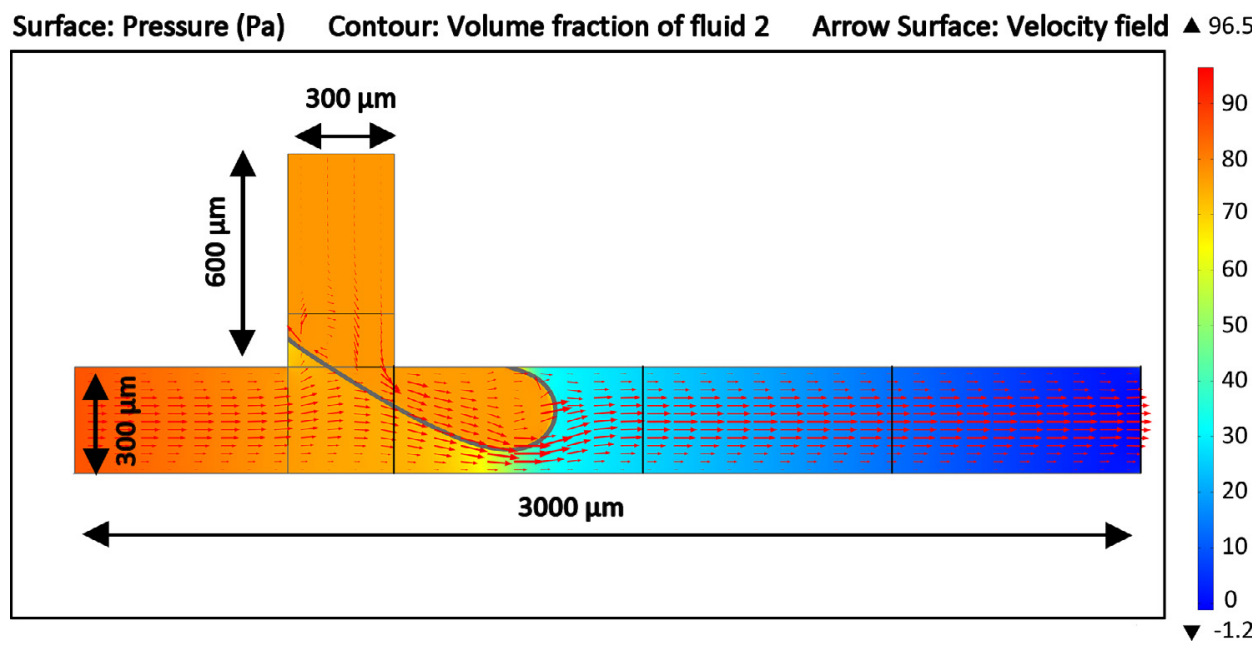

Fig. 7. COMSOL simulation illustrating formation of the droplet. 
Table 2

Equivalent circuit parameters.

\begin{tabular}{|c|c|c|c|c|c|}
\hline Parameter & Tubing length $(\mathrm{m})$ & Oil resistance $\left(\mathrm{Pa} \mathrm{s} / \mathrm{m}^{3}\right)$ & Oil capacitance $\left(\mathrm{m}^{3} / \mathrm{Pa}\right)$ & Water resistance $\left(\mathrm{Pa} \mathrm{s} / \mathrm{m}^{3}\right)$ & Water capacitance $\left(\mathrm{m}^{3} / \mathrm{Pa}\right)$ \\
\hline \multirow{2}{*}{ Fluid tubing $\left(\mathrm{R}_{\mathrm{t}}, \mathrm{C}_{\mathrm{t}}\right)$} & 0.20 & $5.3 \times 10^{11}$ & $1.17 \times 10^{-13}$ & $1.06 \times 10^{10}$ & $1.17 \times 10^{-13}$ \\
\hline & 1 & $26.5 \times 10^{11}$ & $5.84 \times 10^{-13}$ & $5.3 \times 10^{10}$ & $5.84 \times 10^{-13}$ \\
\hline Air tubing $\left(\mathrm{R}_{\mathrm{at}}, \mathrm{C}_{\mathrm{at}}\right)$ & 1.5 & $3.73 \times 10^{5}$ & $5.1 \times 10^{-9}\left(\mathrm{P}_{\mathrm{s}}=9500 \mathrm{~Pa}\right)$ & $3.73 \times 10^{5}$ & $8.73 \times 10^{-9}\left(P_{s}=5500 \mathrm{~Pa}\right)$ \\
\hline Reservoir $\left(C_{a}\right)$ & $\mathrm{N} / \mathrm{A}$ & Negligible & $1.53 \times 10^{-9}\left(\mathrm{P}_{\mathrm{s}}=9500 \mathrm{~Pa}\right)$ & Negligible & $2.64 \times 10^{-9}\left(P_{s}=5500 \mathrm{~Pa}\right)$ \\
\hline Microchannel $\left(R_{c}, C_{c}\right)$ & $\mathrm{N} / \mathrm{A}$ & $78 \times 10^{12}$ & $1.4 \times 10^{-15}$ & $1.56 \times 10^{12}$ & $1.4 \times 10^{-15}$ \\
\hline
\end{tabular}

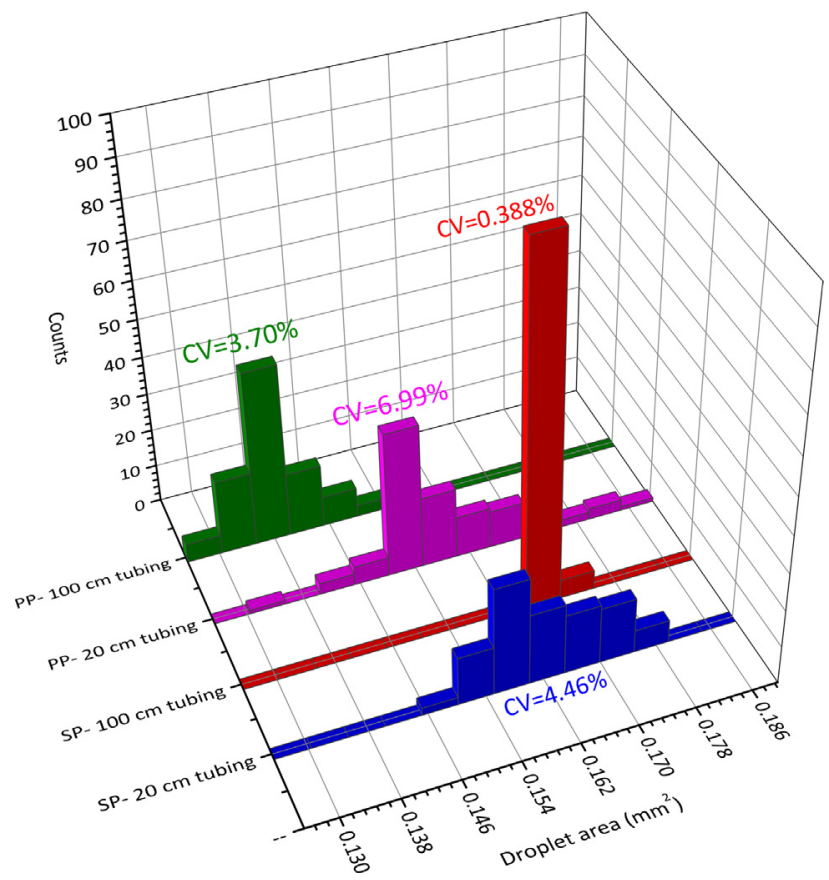

Fig. 8. Histogram of the droplet area obtained experimentally for 100 droplets using two different tubing lengths for syringe pump (SP) and pressure pump (PP) driven systems.

the reservoir play a role in fluctuation damping. Therefore, PPdriven systems inherently operate using such an enhanced compliance damping mechanism.

Fig. 9 summarizes the results obtained using the numerical simulations. When the flow source fluctuations were neglected, very low CV values were obtained $(0.139 \%$ and $0.041 \%)$, which do not match the experimental results. On the other hand, considering the flow source fluctuations and using the methodology explained in the previous section, a strong correlation was obtained between the numerical model and the experimental results. As shown in Fig. 9, in the SP and PP cases, when 20-cm tubing was used, droplet area CV was $0.49 \%$ and $7.45 \%$, respectively. Using $100 \mathrm{~cm}$ of the fluid tubing in the microfluidic system results in a narrower distribution of the droplet area. The CV of the droplet area using $100-\mathrm{cm}$ fluid tubing for the SP and PP cases is $0.175 \%$ and $5.06 \%$, respectively. The decrease in distribution and CV of the droplet area confirms the significance of the effect of tubing length on droplet monodispersity.

It should be noted that for the COMSOL simulations, a much shorter microfluidic channel was analyzed for computational efficiency. Therefore, we do not expect to obtain the exact same results obtained in the experiments. However, numerical simulation results provide the means to analyze the behavior of the system. In addition, the CV values given in Fig. 9 demonstrate the importance of including inlet fluctuations for more realistic numerical models. The comparison between fluctuation-free results for the SP and PP cases shows that syringe pump is advan-

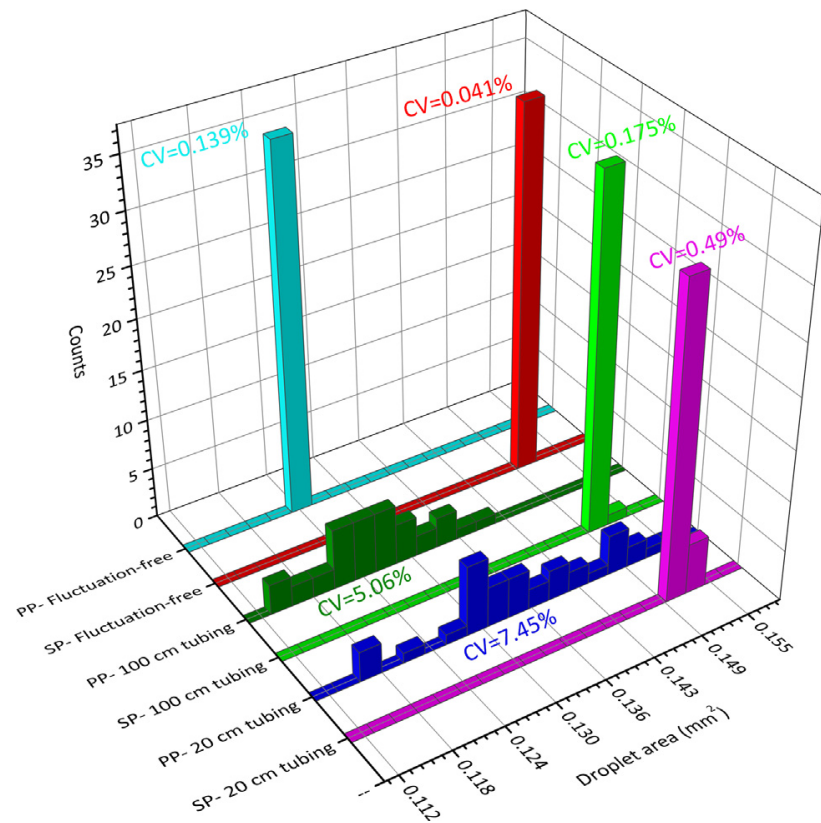

Fig. 9. Histogram of the droplet area obtained numerically for 36 droplets in three conditions of numerical simulation for SP and PP cases.

tageous for generating monodisperse droplets. This is because syringe pump is immune to the internal pressure fluctuations caused, due to droplet generation dynamics and the motion of the droplet inside the microchannel. Additionally, the comparison of experimental (Fig. 8) and numerical (Fig. 9) results reflects the importance of considering the flow source fluctuations. As seen, the numerical results obtained using fluctuation-free inlet conditions are much lower than the experimental results. Once inlet fluctuations were added, a satisfactory correlation was obtained. Furthermore, it is shown that by increasing the off-chip compliance with increased tubing length upstream the channel inlet, significant improvement can be achieved, especially for the syringe pumpdriven system. Fig. 10 shows our experimental droplet monodispersity results in more detail.

As seen from the experimental measurements of individual droplet lengths, increasing the tubing length from $20 \mathrm{~cm}$ to $100 \mathrm{~cm}$ has a remarkable effect on droplet monodispersity. The effect of longer fluid tubing on droplet monodispersity in the syringe pump case (Fig. 10a) is higher than the pressure pump case (Fig. 10b). The insets show the difference in dimensionless form. These results challenge the monodispersity results obtained in the literature, which generally state that pressure pumps perform better than syringe pumps. Our approach shows that such pump comparisons are not conclusive, since the results not only depend on build quality, hence the brand of the pumps, but also on off-chip parts, which are often neglected and have a significant effect on the system. Thus, the microfluidic system should be designed considering both the on-chip and off-chip components and their hydrodynamic damping effects, in order to obtain higher droplet monodispersity. 

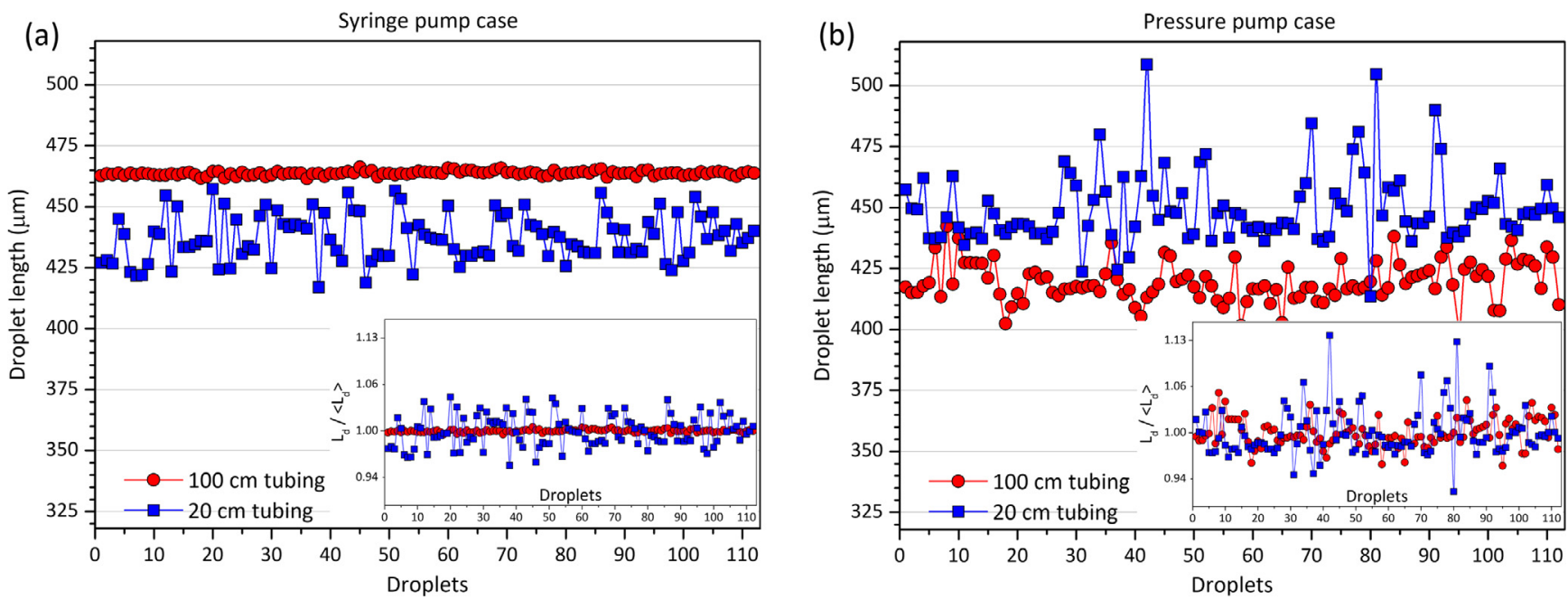

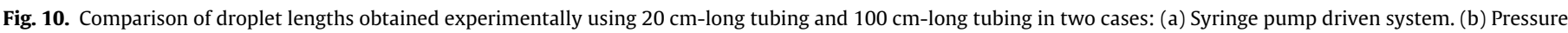
driven system. The insets show the normalized droplet lengths, $\mathrm{L}_{\mathrm{d}}$.

\section{Conclusion}

In this study, we investigated the effect of elastomeric tubing as an off-chip compliant component to damp the hydrodynamic fluctuations caused by external sources. The experiments were conducted using five different lengths of tubing and the hydrodynamic fluctuations were analyzed. The results demonstrate that flow stability inside the microfluidic systems can be increased substantially by using a soft elastomeric tubing as an off-chip compliant component. By increasing the length of the tubing that connects the flow source to the microchip, flow source fluctuations can be damped significantly. The comparison between the syringe and pressure pump-driven systems demonstrates that damping effect in the syringe pump-driven system is more significant, since the pressure pump inherently makes use of a similar compliance effect. To demonstrate the importance of hydrodynamic damping, a model microdroplet generation system was used. We have shown that inlet fluctuation damping improves the monodispersity of microdroplets. Our experimental and numerical results indicate that this effect can be used for droplet generation systems that are driven by either pressure or syringe pumps. It is demonstrated that pressure pumps operate with an inherent damping mechanism, which explains the better monodispersity results obtained in the literature so far. The hydrodynamic damping method presented here is very effective and yields a significant improvement in $\mathrm{CV}$ of droplet area for syringe pump-driven microdroplet systems.

\section{Acknowledgements}

This project was supported by the Scientific and Technological Research Council of Turkey (TÜBITAK, Project No. 215E086). Dr. Elnaz Alizadeh Haghighi is thankful to the support of TÜBITAK BIDEB-2216 Postdoctoral Research Fellowship. The authors also thank Dr. Amar S. Basu for providing DMV software.

\section{References}

Bashir, S., Rees, J.M., Zimmerman, W.B., 2011. Simulations of microfluidic droplet formation using the two-phase level set method. Chem. Eng. Sci. 66, 47334741.

Basu, A.S., 2013. Droplet morphometry and velocimetry (DMV): a video processing software for time-resolved, label-free tracking of droplet parameters. Lab Chip 13, 1892-1901.
Beer, N.R., Rose, K.A., Kennedy, I.M., 2009. Observed velocity fluctuations in monodisperse droplet generators. Lab Chip 9, 838-840.

Boy, D.A., Gibou, F., Pennathur, S., 2008. Simulation tools for lab on a chip research: advantages, challenges, and thoughts for the future. Lab Chip 8, 1424-1431.

Bruus, H., 2007. Theoretical Microfluidics. Oxford University Press, Oxford.

Cartas Ayala, M.A., 2013. Hydrodynamic Resistance and Sorting of Deformable Particles in Microfluidic Circuits. Massachusetts Institute of Technology.

Garstecki, P., Fuerstman, M.J., Stone, H.A., Whitesides, G.M., 2006. Formation of droplets and bubbles in a microfluidic T-junction-scaling and mechanism of break-up. Lab Chip 6, 437-446.

Glawdel, T., Elbuken, C., Ren, C.L., 2012a. Droplet formation in microfluidic Tjunction generators operating in the transitional regime. I. Experimental observations. Phys. Rev. E 85, 016322.

Glawdel, T., Elbuken, C., Ren, C.L., 2012b. Droplet formation in microfluidic Tjunction generators operating in the transitional regime. II. Modeling. Phys. Rev. E 85, 016323.

Glawdel, T., Ren, C.L., 2012. Global network design for robust operation of microfluidic droplet generators with pressure-driven flow. Microfluidics Nanofluidics 13, 469-480.

Hwang, S.-J., Moon, S.-K., Kim, S.E., Kim, J.H., Choi, S.-W., 2014. Production of uniform emulsion droplets using a simple fluidic device with a peristaltic pump. Macromol. Res. 22, 557-561.

Kang, Y.J., Yang, S., 2012. Fluidic low pass filter for hydrodynamic flow stabilization in microfluidic environments. Lab Chip 12, 1881-1889.

Kim, Y., Kuczenski, B., LeDuc, P.R., Messner, W.C., 2009. Modulation of fluidic resistance and capacitance for long-term, high-speed feedback control of a microfluidic interface. Lab Chip 9, 2603-2609.

Korczyk, P.M., Cybulski, O., Makulska, S., Garstecki, P., 2011. Effects of unsteadiness of the rates of flow on the dynamics of formation of droplets in microfluidic systems. Lab Chip 11, 173-175.

Lee, C.-Y., Lin, Y.-H., Lee, G.-B., 2009. A droplet-based microfluidic system capable of droplet formation and manipulation. Microfluidics Nanofluidics 6, 599-610.

Lee, J., Rahman, F., Laoui, T., Karnik, R., 2012. Bubble-induced damping in displacement-driven microfluidic flows. Phys. Rev. E 86, 026301.

Li, C., Xu, J., Ma, B., 2015. A self-powered microfluidic monodispersed droplet generator with capability of multi-sample introduction. Microfluidics Nanofluidics 18, 1067-1073.

Li, Z., Mak, S.Y., Sauret, A., Shum, H.C., 2014. Syringe-pump-induced fluctuation in all-aqueous microfluidic system implications for flow rate accuracy. Lab Chip $14,744-749$.

Link, D.R., Anna, S.L., Weitz, D.A., Stone, H.A., 2004. Geometrically mediated breakup of drops in microfluidic devices. Phys. Rev. Lett. 92, 054503.

Oh, K.W., Lee, K., Ahn, B., Furlani, E.P., 2012. Design of pressure-driven microfluidic networks using electric circuit analogy. Lab Chip 12, 515-545.

Pang, Y., Kim, H., Liu, Z., Stone, H.A., 2014. A soft microchannel decreases polydispersity of droplet generation. Lab Chip 14, 4029-4034.

Raj, A., Halder, R., Sajeesh, P., Sen, A., 2016. Droplet generation in a microchannel with a controllable deformable wall. Microfluidics Nanofluidics 20, 1-16.

Ruzicka, J., Marshall, G.D., Christian, G.D., 1990. Variable flow rates and a sinusoidal flow pump for flow injection analysis. Anal. Chem. 62, 1861-1866.

Schmid, S.R., Hamrock, B.J., Jacobson, B.O., 2014. Fundamentals of Machine Elements: SI Version. CRC Press.

van Steijn, V., Kleijn, C.R., Kreutzer, M.T., 2010. Predictive model for the size of bubbles and droplets created in microfluidic T-junctions. Lab Chip 10, 25132518.

Van Steijn, V., Kreutzer, M., Kleijn, C., 2008. Velocity fluctuations of segmented flow in microchannels. Chem. Eng. J. 135, S159-S165. 
Wang, X., Riaud, A., Wang, K., Luo, G., 2015. Pressure drop-based determination of dynamic interfacial tension of droplet generation process in $\mathrm{T}$-junction microchannel. Microfluidics Nanofluidics 18, 503-512.

Xu, J., Li, S., Tan, J., Luo, G., 2008. Correlations of droplet formation in T-junction microfluidic devices: from squeezing to dripping. Microfluidics Nanofluidics 5 , 711-717.

Xu, J., Li, S., Tan, J., Wang, Y., Luo, G., 2006a. Preparation of highly monodisperse droplet in a T-junction microfluidic device. AIChE J. 52, 3005-3010.
Xu, J., Luo, G., Li, S., Chen, G., 2006b. Shear force induced monodisperse droplet formation in a microfluidic device by controlling wetting properties. Lab Chip 6 , $131-136$.

Zeng, W., Jacobi, I., Beck, D.J., Li, S., Stone, H.A., 2015a. Characterization of syringepump-driven induced pressure fluctuations in elastic microchannels. Lab Chip $15,1110-1115$.

Zeng, W., Jacobi, I., Li, S., Stone, H.A., 2015b. Variation in polydispersity in pump-and pressure-driven micro-droplet generators. J. Micromech. Microeng. 25, 115015. 